\title{
Gobiernos, administraciones y ciudadanía en red
}

\author{
Mentxu RAMILO ARAUJO \\ Profesional in(ter)dependiente \\ mentxu@gmail
}

Recibido: $13 / 01 / 2014$

Aceptado: 11/04/2014

\section{Resumen}

Este artículo hace un repaso a herramientas tecnológicas disponibles en Internet, que muchas organizaciones, empresas y personas ya están utilizando; y que ofrecen numerosas posibilidades a las instituciones públicas para ser sociables, trabajar en red y elaborar políticas públicas y servicios públicos contando con la ciudadanía.

Siempre que exista voluntad humana (política, directiva, técnica) diversas tecnologías pueden facilitar la información, la consulta, la conversación y el diálogo, la deliberación y la colaboración entre las instituciones públicas y los agentes públicos, privados y sociales, claves para lograr el éxito de las políticas y servicios públicos.

Palabras clave: Instituciones públicas, ciudadanía, Internet, medios sociales, gobierno relacional.

\section{Governments, administrations and citizenship in red}

\begin{abstract}
This article gives an overview of technological tools available on the Internet, which many organizations, businesses and individuals are already using. These tools offer numerous possibilities to public institutions to be sociable, to network and develop public policies and public services taking into account citizens.

Whenever there is human will (political, managerial, technical) various technologies can provide information, consultation, conversation and dialogue, deliberation and collaboration among public institutions and the public, private and social stakeholders for the success of public policies and public services.
\end{abstract}

Key words: Public institutions, citizens, Internet, social media, governance.

\section{Referencia normalizada}

Ramilo Araujo, M. (2014). "Gobiernos, administraciones y ciudadanía en red". Cuadernos de Gobierno y Administración Pública, Vol 1, Núm. 1.: 77-96.

Sumario: Introducción. 1. Instituciones públicas para el Siglo XXI. 2. Uso eficiente y con sentido de las TIC: ¿medios o fines?. 3. Niveles de utilización de las TIC. 3.1. Información a la ciudadanía. 3.1.1. Páginas web: Presencia en internet. 3.1.2. Información proactiva. 3.1.3. Acceso, simplificación y visualización de la información. 3.2. Consulta ciudadana. 3.2.1. Llamada telefónica. 3.2.2. Mensajería instantánea. 3.2.3. Correo electrónico. 3.2.4. Formularios en línea. 3.2.5. Foros de participación. 3.3. Conversar y dialogar. 3.3.1. Blogs. 3.3.2. Redes Sociales. 3.4. Deliberación y colaboración. 3.4.1. Herramientas para trabajar colaborativamente. 3.4.2. Wikis. 4. Ciudadanía y la sociedad civil en red. 5. Reflexiones finales. 6. Bibliografía. 


\section{Introducción}

En los últimos años numerosos paradigmas han analizado el impacto que las Tecnologías de la Información y la Comunicación (en adelante TIC) pueden tener sobre las instituciones públicas y en su interacción con la ciudadanía. Muchos libros, artículos, jornadas, conferencias y proyectos se han desarrollado sobre la administración electrónica, el gobierno electrónico, la democracia electrónica o recientemente, las iniciativas de gobierno abierto (Barber, 2001; Criado, Ramilo, y Salvador, 2002; Criado y Ramilo, 2001; Hale, Musso, y Weare, 1999; Hofmann, Ramirez, y Bojórquez, 2012; Ramilo, 2003; White House, 2009). Quizá, hasta el momento, haya sido mayor la reflexión, la producción académica y el gasto tecnológico en estos proyectos de moda, que la concreción, comprensión y uso efectivo de estas tecnologías por parte de las instituciones pública y de la ciudadanía.

Por otro lado, las apuestas por la apertura de las instituciones públicas y la creencia de que en el siglo XXI la legitimidad institucional dependerá de una ciudadanía activa (in)formada e interesada por lo público, difícilmente encajan con el uso interesado de la información personal y privada por parte de las grandes empresas tecnológicas y de algunos gobiernos occidentales. Las prácticas de espionaje ocultadas, alegando razones de seguridad, paralelas al discurso de transparencia y apertura, erosionan la construcción de redes de comunicación y de sociedades libres y democráticas. En pleno siglo XXI, la libertad de expresión y el acceso libre a información pública deberían asegurarse y ser derechos de ciudadanía. Sin embargo, las TIC no son neutrales y ampliar o limitar sus posibilidades de uso, dependerá de los valores y de los propósitos que definan las organizaciones y las personas que las utilicen (Estebaranz y Ramilo, 2013).

\section{Instituciones públicas para el Siglo XXI}

La ciudadanía frecuentemente aparece como destinataria de los proyectos de reforma, modernización e innovación de las instituciones públicas. Pero, ¿realmente se le consulta para conocer cuáles son sus necesidades y preferencias? ¿O se intuyen a partir de estudios procedentes generalmente de sectores empresariales en los que prima, desde luego, el rol de cliente o persona usuaria más que el de ciudadanía activa?

Veintiséis años después del informe de la OCDE "La Administración al servicio del cliente" (OCDE, 1987), la Comisión para la Reforma de las Administraciones Públicas (CORA, 2013) volvió a plantear la necesidad de racionalizar estructuras, procedimientos y recursos públicos. ¿Qué ha pasado en estos años? ¿Por qué les cuesta tanto a las instituciones públicas poner, verdaderamente, a la ciudadanía en el centro de la acción política y pública? Porque hablamos de principios y valores; hablamos de personas, de voluntades, de intereses y de percepciones.

Si la vocación de servicio público y la orientación a la ciudadanía no forman parte del código genético de las personas que toman decisiones en las instituciones públicas, por muchos cambios de fachada, uso de tecnologías innovadoras y discursos de moda que se hagan (eAdministración, eGobierno, Gobierno2.0, Gobierno Abierto, etc), el corazón de las organizaciones seguirá latiendo desde una lógica competencial jurídico-administrativa; muy 
alejada de una filosofia de política y de gestión pública para (y con) la ciudadanía (Criado y Ramilo, 2001; Graells y Ramilo, 2013; Ramilo, 2005).

La mentalidad de las personas que trabajan en las instituciones públicas ha de avanzar desde el control a la confianza; y de la visión procedimental a una mayor autonomía. Es necesario pasar de organizaciones basadas en las normas y competencias a organizaciones basada en las personas y la resolución de problemas. Y, en lugar de rígidas estructuras jerárquicas, es fundamental avanzar hacia organizaciones que trabajen en red con mayor apertura, dinamismo y agilidad. Estas transformaciones permitirán avanzar hacia Gobiernos, Administraciones y ciudadanía en red (Graells y Ramilo, 2013; Ortíz, 2009).

Facilitar el acceso a la información relevante y comprensible de las actuaciones públicas, escuchar a la ciudadanía, generar sinergias y tejes redes de colaboración, actuar de manera estratégica, o reconocer y visibilizar el trabajo de sus profesionales, entre otras, podrían ser algunas de las claves para lograr la racionalización y sostenibilidad de las administraciones públicas y, mucho más importante aún, para lograr recuperar la confianza de la ciudadanía en las instituciones públicas y lograr, de esta manera que la ciudadanía, los gobiernos y administraciones públicas interactúen en armonía por el bien común y poniendo en valor los recursos públicos (Graells y Ramilo, 2013).

En todo este proceso transformador, las TIC pueden ser motores de cambio, siempre que haya voluntad política y directiva transformadora para utilizar las herramientas de manera eficiente, con sentido y sacándoles el mayor provecho posible.

\section{Uso eficiente y con sentido de las TIC: ¿medios o fines?}

Son amplias y variadas las posibilidades tecnológicas para que la ciudadanía pueda (in)formarse, interactuar y tomar parte en la vida política, siempre que exista voluntad política que concrete para qué quieren las instituciones públicas relacionarse e interactuar con la ciudadanía, con qué objetivos, y para lograr qué fines.

Las tecnologías están desarrolladas. Es una cuestión de voluntad política y pública abrir (o no) los procesos de decisión para tener en cuenta las voces diversas de la ciudadanía. Desde las instituciones públicas es fundamental animar a la ciudadanía, reconocer y poner en valor su participación, ya que cuando la ciudadanía decide tomar parte en procesos participativos, dedica sus recursos (tiempo, conocimientos, dinero, etc.) al servicio de la sociedad (Reig, 2012; Subirats, 2011).

Si las instituciones públicas y la ciudadanía utilizasen de manera efectiva y con sentido algunas de las numerosas herramientas tecnológicas ya disponibles en Internet, entre otras cuestiones, podrían:

- relacionarse con mayor transparencia, eficacia y eficiencia;

- ampliar las posibilidades de participación e implicación ciudadana en la vida política y pública;

- mejorar las políticas públicas y los servicios públicos;

- conseguir mejorar el rendimiento de las instituciones públicas y de los recursos públicos; 
- responder mejor y a tiempo a las necesidades de sociedades cada vez más complejas que demandan respuestas y servicios personalizados;

- y en definitiva, recuperar la legitimidad de las instituciones públicas y poner en valor los bienes comunes y públicos.

Sin embargo, desgraciadamente, en numerosas ocasiones, la inversión en TIC se ha convertido en un fín en sí misma: se gasta en tecnología sin abordar el cambio de mentalidad de las personas (motivación, formación, competencias); sin modificar el funcionamiento de las organizaciones y de los procesos de trabajo (creando entidades electrónicas paralelas); y sin utilizar con sentido y de manera eficiente el despliegue tecnológico realizado.

De esta manera, el verdadero fin de la incorporación de las TIC en las instituciones públicas, que es lograr su transformación (simplificación, interoperabilidad, agilidad, proactividad en la prestación de servicios, transparencia, procesos participativos, rendición de cuentas, etc.) se ve muy limitado. En numerosas ocasiones, ni las propias personas trabajadoras en las instituciones públicas ni la ciudadanía, perciben mejoras. Al contrario, se frustran, ya que a pesar de las posibilidades tecnológicas existentes y las inversiones realizadas en tecnología, el funcionamiento de las instituciones públicas sigue siendo lento e ineficiente.

\section{Niveles de utilización de las TIC}

Este artículo propone una serie de niveles de utilización de las TIC por parte de las instituciones públicas para interactuar con la ciudadanía. En cada nivel (información, consulta, conversar y dialogar, deliberación y colaboración) se mencionan algunas herramientas útiles, porque permiten crear e intercambiar contenidos de manera rápida, sencilla y a un bajo coste. A estas herramientas se les ha denominado herramientas web2.0 o también webs o medios sociales (O'Reilly, 2005).

Una de las claves del éxito de estos medios sociales es la facilidad y la rapidez para crear contenidos, compartir información, conversar o interactuar de manera colaborativa con otras personas en Internet. Frente a los medios de comunicación de masas (mass media) donde la comunicación es unidireccional; los medios sociales permiten a cualquier persona generar y emitir información y contenidos; e interactuar con todas aquellas personas con las que quiera conversar (Reig, 2012)

La facilidad de uso de los medios sociales ha convencido a gran parte de la población mundial para hacer de estas herramientas su espacio de conversación e interacción. Son en definitiva, medios sociables. Sin embargo, las instituciones públicas tienen más dificultades para encontrar ventajas y oportunidades en estos nuevos medios sociales de comunicación (Estebaranz y Ramilo, 2013; F. Barrero, Criado, y Ramilo, 2006). Para acercar estas herramientas a la realidad de las instituciones públicas, de cada una de ellas se indicarán algunas ventajas e inconvenientes de su uso y algunos ejemplos reales de utilización. 


\subsection{Información a la ciudadanía}

Cuando la ciudadanía decide (in)formarse para ejercer sus derechos y obligaciones con las instituciones públicas utilizando las TIC podría: acceder a las páginas web de las instituciones públicas; suscribirse por RSS para obtener información de forma proactiva; recibir información accesible, comprensible y que se visualice fácilmente.

\subsubsection{Páginas web: Presencia en internet}

La evolución de las páginas web de las instituciones públicas ha sido espectacular en los últimos diez años. Han dejado de ser escaparates estáticos, con escasa información actualizada y sin apenas servicios en línea; para convertirse en lugares dinámicos que integran información de seguimiento de la actividad política y pública (plenos, normativa, políticas, eventos), servicios públicos electrónicos, información proactiva o espacios para la participación (desde los más sencillos, como el correo electrónico para enviar sugerencias y reclamaciones, a la presencia de formularios en línea, foros, etc.) (Borge, Colombo, y Welp, 2008; Criado y Ramilo, 2001; Ramilo, 2003; Salvador, Cortés, Sánchez, y Ferrer, 2004).

Sin embargo, a pesar de los esfuerzos realizados en los últimos años para digitalizar los servicios públicos, el nivel de utilización de los mismos sigue siendo escaso (ONTSI, 2013) ${ }^{1}$. Entre las causas que explicarían esta brecha entre la oferta de servicios públicos electrónicos y la demanda de los mismos, podrían señalarse, entre otras: la brecha digital, el desconocimiento de la existencia de servicios telemáticos, las dificultades (técnicas, formativas, culturales) para tramitar en Internet, la falta de confianza en los canales telemáticos.

Crear una página web dinámica con información actualizada y proactiva requiere mucha dedicación y tiene un coste elevado, a menos que se pongan en marcha iniciativas como la del Ayuntamiento de Nueva York ${ }^{2}$ (Reinventar NYC.gov) para mejorar la web y sus servicios contando con el trabajo colaborativo de distintos profesionales en el diseño, desarrollo y lanzamiento de un nuevo portal web de la ciudad con una mayor orientación a la ciudadanía (Guadián, 2011a).

La ciudadanía cada día es más crítica y exigente con las instituciones públicas. Reclama transparencia y responsabilidad en la inversión del dinero público. Los proyectos tecnológicos suelen costar mucho dinero. Y antes de inaugurarse, suelen estar ya desfasados. Siempre existen voces críticas que consideran que utilizando software de fuentes abiertas se podrían ahorrar muchos costes en licencias y reutilizarlos en otras inversiones. Además es importante dejar constancia de qué empresas, cuántas personas y por cuánto tiempo han trabajado en los proyectos tecnológicos y los presupuestos desglosados para poder exigir responsabilidades. De esta manera,

\footnotetext{
${ }^{1}$ Nivel de digitalización de los servicios: Administración General del Estado (93\%); Comunidades Autónomas (80\%) y Entes Locales (70\%). El 44,7\% de la ciudadanía utiliza servicios públicos electrónicos (ONTSI 2013: 177 y 194).

${ }^{2}$ www.nyc.gov
} 
será posible auditar los excesos invertidos en tecnología, como por ejemplo, sucedió con la web del Senado [www.senado.es] que le costó a la ciudadanía española 450.000 euros y requirió casi un año de trabajo de tres equipos de personas expertas mientras que una sola persona, en una semana, con 0 euros en licencias, hizo una réplica de la web [www.senado.3a2.com] (Bosco y Caldana, 2012).

\subsubsection{Información proactiva}

La proactividad es una de las características más importantes de los nuevos servicios y herramientas disponibles en Internet. Que las instituciones públicas informen de forma proactiva a la ciudadanía sobre temas de su interés es un gran avance. Algunas informaciones sobre las que se habilita la suscripción suelen ser la agenda, noticias, avisos, ofertas de empleo, ayudas y subvenciones, contrataciones públicas, transporte público, etc.

La aparición de servicios de suscripción a información, a día de hoy, hace pensar en posibles servicios personalizados o a la carta para que, por ejemplo, las instituciones públicas nos informen, por el canal que escojamos (SMS, e-mail, RSS, redes sociales, etc) sobre aquellos temas concretos de nuestro interés.

Las páginas web que tienen la opción de suscripción por medio de RSS (en inglés really simple syndication), normalmente mostrando un icono similar a ésteß, permiten la suscripción de forma muy sencilla y gratuita a la información nueva que se publica en esas páginas web. El Boletín Oficial del Estado (BOE), que desde enero de 2009 no se publica en papel, ha mejorado mucho la forma de acceder, consultar y encontrar la información. Ofrece la posibilidad de suscribirse por medio de RSS a las distintas secciones del Boletín o bien a canales temáticos. Puede accederse a las suscripciones desde: www.boe.es/rss.

La ventaja principal es que podemos agrupar fuentes de información RSS de diferentes webs y recibir los contenidos nuevos en cuanto se produzcan en un solo lugar (denominado agregador o lector de noticias), ahorrándonos visitar distintas web para ver si hay noticias nuevas. Bloglines.com, Google Currents, Feedly.com, Flipboard, Friendfeed.com o Netvibes.com son algunos de los lectores más utilizados desde distintos dispositivos, sean computadoras tradicionales, smartphones o tabletas. Al recibir la información en formato digital, puede reutilizarse, citarse, compartirse, analizarse, mejorarse, dándole mayor valor. El inconveniente más importante es el desconocimiento de esta tecnología RSS y cómo utilizar un lector de noticias.

\subsubsection{Acceso, simplificación y visualización de la información}

Respecto a la información pública, básica para que la ciudadanía pueda documentarse y formarse un criterio a la hora de participar en la vida política, es necesario seguir haciendo esfuerzos para garantizar el acceso a la misma (planes de gobierno, memorias, contratos, presupuestos, procedimientos, expedientes, etc), simplificar su presentación y lenguaje y mejorar su visualización. 
Algunas iniciativas de apertura de datos públicos a destacar, podrían ser: el distrito de Columbia ${ }^{3}$, el proyecto Data.gov del Gobierno de los Estados Unidos, el proyecto OD4D - Open Data for Development de América Latina y el Caribe, el Portal de datos de la Comisión Europea ${ }^{5}$, Datos.gob.es para el conjunto del Estado español, el proyecto Open Data Euskadi ${ }^{6}$ del Gobierno Vasco o Dades Obertes ${ }^{7}$ de la Generalitat de Cataluña.

Estas iniciativas pretenden poner a disposición de la ciudadanía, empresas, asociaciones, centros de investigación, etc. datos públicos que puedan ser reutilizables. De esta manera, podrán crearse aplicaciones y servicios derivados de los mismos. Los conjuntos de datos expuestos se ofrecen bajo licencias de propiedad abiertas, que permiten su redistribución, reutilización y aprovechamiento con fines comerciales.

Se han puesto en marcha concursos dirigidos a la promoción de la apertura y reutilización de la información pública, como Apps for Democracy ${ }^{8}$ en Estados Unidos, Open Data Challenge en la Unión Europea o Desafio Abredatos ${ }^{10}$ en España. Estas iniciativas pretenden animar a personas emprendedoras, investigadoras y a empresas a desarrollar servicios y aplicaciones a partir de datos liberados. Es interesante analizar el impacto económico que pueden suponer este tipo de iniciativas. El concurso Apps for Democracy, puesto en marcha en el año 2008 por el distrito de Columbia, fue pionero en liberar más de 480 bases de datos públicos e impulsó la creación de aplicaciones (apps) que aportaran valor. La iniciativa costó $50.000 \$$ y la estimación del valor de las apps creadas fue de 2.300.000\$.

En segundo lugar, es también importante simplificar el lenguaje administrativo para hacer comprensible la información pública. Sin olvidar en ningún momento que un lenguaje simple y claro no implica que sea menos riguroso, veraz y legal. Además, resulta fundamental racionalizar y hacer accesible el lenguaje administrativo; elaborar textos claros, correctos, usando un lenguaje inclusivo y que sean visualmente atractivos, tanto si son en papel como en formato electrónico.

En tercer lugar, resulta de interés mejorar la visualización de la información pública para hacerla atractiva y no infoxicar o sobrecargar a la ciudadanía con documentos extensos e indescifrables. Al garantizar el acceso a la información pública y permitir su reutilización, es posible que surjan nuevos servicios que aporten mayor valor a las propias administraciones públicas, a la ciudadanía y las empresas. Esta información enriquecida deberá, al mismo tiempo, seguir siendo accesible.

\footnotetext{
${ }^{3} \mathrm{http}: / /$ data.dc.gov

${ }^{4}$ www.od4d.org

${ }^{5}$ http://open-data.europa.eu/open-data/es/

${ }^{6} \mathrm{http}$ ://opendata.euskadi.net

${ }^{7}$ www20.gencat.cat/portal/site/dadesobertes

${ }^{8}$ www.appsfordemocracy.org

${ }^{9}$ http://opendatachallenge.org

${ }^{10}$ https://es.twitter.com/abredatos
} 
Estos nuevos servicios, seguramente usen técnicas de visualización que permitan simplificar la complejidad de los datos. Algunas iniciativas interesantes de visualización de la información pública, pueden ser Gapminder ${ }^{11}$, sitio web que recopila numerosas bases de datos de información pública de distintos estados y organismos de Naciones Unidas para visualizarlos de forma atractiva y sencilla por medio de vídeos y animaciones con la evolución de diferentes datos de países y regiones de todo el mundo.

Las animaciones son interactivas y uno puede elegir y comparar los datos de aquellos países que se deseen visualizar. El directorio de Google Public Data ${ }^{12}$ se puede ver, por ejemplo, con la tecnología de animación estadística de Gapminder que permite visualizar gran cantidad de datos de una manera muy didáctica. La Fundación Gapminder, que promueve el desarrollo sostenible mundial mediante una mayor utilización y entendimiento de las estadísticas sobre el desarrollo social, económico y ambiental a nivel local, nacional y mundial.

Las infografias ${ }^{13}$ y los mapas interactivos también pueden ser herramientas idóneas para sintetizar mucha información en poco espacio y visualizarla a un coste bajo de tiempo y esfuerzo. Otros ejemplos de temáticas sobre las que mejorar la visualización de la información pueden ser: permitir a la ciudadanía conocer el destino de los impuestos $^{14}$, conocer las licitaciones y contratos que hay en marcha y opinar sobre ellos $^{15}$, informarse en tiempo real de la situación financiera de un país ${ }^{16}$, o visualizar las transacciones económicas a nivel mundia ${ }^{17}$. Todo ello sin tener que leer extensísimos documentos, sino accediendo a una web donde la información se presenta de forma sintética y pedagógica. Por ello en la sociedad en la que vivimos, tanto el acceso a la información relevante como su correcta visualización son fundamentales en cualquier proceso de toma de decisiones, para que podamos participar con conocimiento de causa (Moraga, 2009).

\subsection{Consulta ciudadana}

Es fundamental que las instituciones públicas escuchen de manera activa por múltiples canales lo que la ciudadanía quiere transmitir, bien para reclamar y quejarse; como para agradecer y aportar ideas o propuestas de mejora para los servicios públicos y políticas públicas. A través de las llamadas telefónicas, la mensajería, el correo electrónico, las encuestas o los foros de participación, la ciudadanía puede consultar a las instituciones públicas y ser consultada por ellas.

${ }^{11}$ www.gapminder.org/data

12 www.google.com/publicdata/directory

${ }^{13} \mathrm{http} / / /$ bit.ly/dY4Dxn

${ }^{14} \mathrm{http}: / /$ dondevanmisimpuestos.es (Presupuestos de la Administración General del Estado y Comunidades Autónomas de España)

${ }^{15} \mathrm{http} / / /$ gastopublico.es o http://comogastan.com

${ }^{16} \mathrm{http}: / /$ usdebtclock.org

${ }^{17} \mathrm{http}$ ://openspending.org 


\subsubsection{Llamada telefónica}

Canalizar las consultas ciudadanas, las quejas, las reclamaciones, las sugerencias y las incidencias a través de un servicio de atención ciudadana telefónica, es una estrategia que han adoptado muchas administraciones públicas. La posibilidad de acceder a este servicio es universal, puesto que toda la población sabe hacer una llamada telefónica.

La atención ciudadana telefónica es un canal ágil para resolver muchas solicitudes de información, comunicar incidencias, e incluso poder tramitar solicitudes con las administraciones públicas. El teléfono 010 en los ayuntamientos, el 012 en las comunidades autónomas y el 060 en la Administración General del Estado son ejemplo de ello.

Si el servicio traslada con rapidez las demandas de la ciudadanía al área correspondiente, es un canal fundamental para conocer las necesidades y problemáticas reales de las personas de la ciudad, región, país. Sin embargo, el coste del personal que atiende estas consultas es elevado y en épocas de crisis, estos servicios de atención directa con la ciudadanía y canalización de sus demandas, están siendo recortados. Para la ciudadanía, es un canal cómodo y fácil de utilizar. Y su coste puede variar según sea una llamada gratuita, tenga un coste reducido de llamada local o sea una llamada de tarificación adicional.

\subsubsection{Mensajería instantánea}

También pueden abrirse canales para enviar a la ciudadanía, a través de SMS, notificaciones, recordatorios, avisos; o permitir a la ciudadanía enviar por medio de SMS sugerencias, reclamaciones, incidencias observadas, etc. El Ayuntamiento de Lleida fue pionero en realizar notificaciones oficiales a través de mensajes de móvil gracias a la operadora local Lleida.net, que creó el sistema SMS certificado ${ }^{18}$, con la misma validez que las cartas certificadas o los burófax.

Podrían utilizarse también otros servicios de mensajería como WhatsApp, ampliamente utilizados por la ciudadanía, para informar y prestar ayuda. Los servicios de emergencia de la Comunidad de Madrid ${ }^{19}$ han utilizado WhatsApp para localizar personas accidentadas gracias al sistema de geolocalización de la herramienta. Por su parte, el Ayuntamiento de Mataró ${ }^{20}$ utiliza WhatsApp como canal de información y atención ciudadana con el mismo horario que el 010.

La ventaja de utilizar estos canales es que la mayor parte de la población tiene a su alcance un teléfono móvil; y cada vez más se está extendiendo el uso de los smartphones. De forma que sería un canal fácil de utilizar y rápido para enviar y recibir comunicaciones.

El inconveniente, en ambos casos, es que cuesta dinero: a la organización porque tiene que reservar un número de teléfono para recibir los mensajes; y a quien participa porque tendrá que pagar por enviar un SMS o por tener una tarifa de datos para acce-

\footnotetext{
${ }^{18} \mathrm{http}: / /$ www.lleida.net/es/servicios/sms-certificado

${ }^{19} \mathrm{http}: / /$ huff.to/Uk9ilg

${ }^{20} \mathrm{http}: / /$ bloc.localret.cat $/ \mathrm{p}=3884$
} 
der a Internet con su smartphone donde no tenga acceso a una red Wi-Fi gratuita. Otro inconveniente puede ser no tener recursos suficientes para atender a las consultas que se planteen por esas vías, si no se concretan bien los casos de uso de ese canal. El Gobierno de Navarra ${ }^{21}$ comenzó a utilizar WhatsApp para la información y atención ciudadana. Pero lo han valorado como un error al no tener capacidad de respuesta por el elevado volumen de peticiones.

\subsubsection{Correo electrónico}

Una posibilidad para que la ciudadanía pueda participar es enviar un mensaje con comentarios, quejas o sugerencias a la dirección electrónica que la Administración pública establezca como canal para la comunicación. Si se conoce la dirección y se tiene acceso a Internet, es una forma sencilla de comunicar y dejar constancia del mensaje enviado. Pero los inconvenientes para gestionar esos mensajes con rapidez y de forma transparente son la brecha en el acceso a Internet y la ausencia de un compromiso institucional con la respuesta. Habrá que concretar quién garantiza que haya una respuesta en modo, forma y tiempo adecuados.

\subsubsection{Formularios en línea}

Herramientas como encuestafacil.com, tusencuestas.com, surveymonkey.com o los formularios de Google Drive son gratuitas y fáciles de usar. También se pueden crear aplicaciones a medida, que tendrán un coste mayor, pero permiten personalizarse, adecuarse a las necesidades concretas y tener mayores garantías de seguridad de los datos recogidos en el formulario.

Los formularios en línea, además de su uso principal para hacer trámites con la Administración de forma ágil, son también otra forma de recabar de modo rápido y sencillo opiniones y valoraciones de la ciudadanía. Desaparecen los costes de imprimir y difundir las encuestas; y de volcar y procesar los datos de forma manual. Crear un formulario en línea es tan sencillo como pensar las preguntas que se quieren realizar, escribirlas utilizando una de las muchas herramientas existentes para crear formularios y difundirlos. Las personas que rellenan el formulario realizan el trabajo de volcar la información en un soporte digital que facilita el posterior tratamiento de los datos. Las ventajas son la sencillez y rapidez tanto para los responsables a la hora de crear, difundir y analizar los resultados del formulario, como para la ciudadanía a la hora de rellenarlo y enviarlo.

El inconveniente principal que presentan los formularios es que hay que tener acceso a Internet. Los usos que se pueden hacer de los formularios son muchos: recogida de ideas, propuestas, quejas y sugerencias; evaluar actividades o servicios; realizar votaciones para priorizar y elegir entre varias propuestas, etc. Pero, mucho cuidado, porque las personas que rellenan un formulario esperan una respuesta. No vale el silencio administrativo.

\footnotetext{
${ }^{21} \mathrm{http}: / /$ bit.ly/wq7qUJ
} 


\subsubsection{Foros de participación}

Los foros pretenden que la voz ciudadana pueda ser oída en los procesos de toma de decisiones. Se han articulado generalmente a través de las Agendas Locales $21^{22}$ que promueven en el ámbito local modelos de desarrollo sostenibles, tratando de contar con la participación de todos los agentes implicados en la vida del municipio.

Estos foros también se han trasladado a Internet y las ventajas son que en cualquier momento una persona puede hacer sus comentarios y aportaciones sobre la temática que le interesa, sin esperar a que se convoque el foro presencial. También puede fomentar el debate e intercambio de ideas. Los inconvenientes son que no se dinamicen lo suficiente por parte de las instituciones públicas y que no se tengan en cuenta las aportaciones volcadas.

\subsection{Conversar y dialogar}

Tras informar y consultar a la ciudadanía, un paso más avanzado es la comunicación bidireccional fluida. En ese intercambio de ideas, opiniones y puntos de vista, pueden surgir propuestas y alternativas de actuación a tener en cuenta en la toma de decisiones políticas. De esta manera, las políticas y servicios públicos, se adecuarían mejor a las necesidades de la ciudadanía. Entre las herramientas que facilitan la interacción con la ciudadanía vamos a analizar los blogs y las redes sociales.

\subsubsection{Blogs}

Los propósitos que han hecho aparecer millones de blogs en los últimos años son múltiples y variados. Conversar, interactuar, conocer opiniones y tener feedback para mejorar, pueden ser algunas motivaciones para responsables políticos y públicos que quieran crear un blog con contenidos de interés.

Una persona con responsabilidades políticas o públicas que pretenda interactuar con la ciudadanía, podría tener un blog donde informar de la actividad política y pública, de los proyectos en marcha o reflexiones personales e interactuar con la ciudadanía por medio de los comentarios en su blog y también conectando su blog con las redes sociales (YouTube, Facebook, Twitter, Flickr, Scridb, SlideShare, LinkedIn o Google+). También hay organizaciones (o programas) que mantienen un blog para interactuar con su público objetivo (Criado y Martínez, 2010).

A continuación se recogen algunos ejemplos de blogs para distintos propósitos:

- Blog de rendición de cuentas: Para tener un punto de vista más personal de los asuntos de la UE, varios comisarios de la Unión Europea y altos cargos de la Comisión en los países miembros tienen sus propios blogs [http://europa.eu/contact/take-part/blogs/index es.htm].

- Blog especializado: Greenversations [http://blog.epa.gov/blog] es el blog de la Agencia Protección Medioambiental de Estados Unidos, para difundir y compar-

${ }^{22}$ http://bit.ly/10uiQSM 
tir conocimientos especializados en temas medioambientales para profesionales que trabajan en ese sector y quieren conocer y compartir información novedosa en la materia.

- Blog de proyecto: Plan de Innovación Pública [http://pip.blog.euskadi.net] su finalidad era compartir y mostrar los hitos, avances y actividades del Plan de Innovación Pública.

- Blog didáctico: USA.gov 「http://blog.usa.gov $\rceil$ tiene como propósito ser un blog que facilite el acceso a la información y a los servicios públicos simplificando y haciendo comprensible el lenguaje administrativo a través de historias y casos de uso.

- Blog de reflexión: Administraciones en Red [http://eadminblog.net] es un blog colaborativo activo desde 2005 creado por dos funcionarios del Gobierno Vasco para reflexionar sobre el papel de las administraciones públicas.

- Blogosfera pública: son todas aquellas personas que en sus blogs y páginas web hablan de, sobre y para las administraciones públicas (Guadián, 2012).

- Bloggers que hacen ciudad: como ejemplo, el espacio para bloggers en la web oficial del gobierno sueco [http://blogs.sweden.se]. La intención es poder mostrar múltiples facetas de Suecia y la cultura sueca por medio de las experiencias y las vivencias de bloggers.

Entre las ventajas de crear y mantener un blog, respecto a una página web estática, se encuentran el hecho de que sea mucho más fácil, económico y rápido actualizar con nuevas noticias un blog que una página web. De hecho, hoy se considera un blog a aquella página web que: se actualiza periódicamente, admite comentarios, puede ser mantenida por personas usuarias que no tienen por qué ser informáticas y tiene servicios que aportan valor añadido como un buscador, traductor, RSS, etc. El lenguaje en los blogs suele ser más informal y, por tanto, es una buena forma de acercar la vida política a la ciudadanía.

Los inconvenientes pueden ser que no se actualice su información, dando a la ciudadanía una sensación de olvido y descuido por parte de las personas autoras del blog (Ramilo y Fernández, 2012).

\subsubsection{Redes Sociales}

Estar donde está la ciudadanía, realizar una escucha activa de sus opiniones sobre las actuaciones públicas y conversar con la ciudadanía pueden ser algunas de las razones más importantes para que las instituciones públicas decidan estar presentes en las redes sociales. Esos espacios virtuales, cada día más utilizados, tienen unas formas de comunicación horizontales diferentes a los entornos jerarquizados de las organizaciones públicas y, por ello, es importante conocer el lenguaje y las formas de interacción de estos nuevos canales de comunicación para poder llegar a la ciudadanía con los mensajes que las instituciones públicas les quieran transmitir (Guadián, 2012; ONTSI, 2011).

Para facilitar y guiar en el diseño de una estrategia de presencia en las redes sociales, varias administraciones públicas han elaborado guías de usos y estilo en las redes sociales (Generalitat de Cataluña, 2013; Gobierno Vasco, 2012; Junta de Casti- 
lla y León, 2012). Una buena práctica puede ser tener en la página web oficial una lista de todos los perfiles públicos oficiales para que la ciudadanía sepa con certeza cuáles son. Un buen ejemplo puede ser el listado de perfiles de los distintos departamentos y organismos públicos de los gobiernos catalán ${ }^{23} \mathrm{y} \mathrm{vasco}^{24}$.

El mundo de las redes sociales no se acaba en Facebook. Hay otras muchas plataformas de interacción social generalistas o temáticas, públicas o privadas que pueden aportar valor a un proceso participativo. Habrá que estar en una, varias o ninguna red social, dependiendo de los contenidos que queramos comunicar o compartir: textos y presentaciones (Scribd, SlideShare, Issuu), imágenes (Flickr, Picasa, Pinterest, Instagram) o vídeos (YouTube, Vimeo). Según quién sea nuestro público objetivo y nuestros propósitos, optaremos por unas u otras redes sociales. Por ejemplo, si se está diseñando un plan de promoción económica y empleo, tal vez haya que crear un grupo de debate en la red profesional LinkedIn para contar con la participación de personas expertas y emprendedoras que tengan ideas interesantes que aportar.

Twitter es una herramienta de participación abierta muy potente para escuchar y dialogar que ofrece muchas posibilidades a las administraciones públicas para interactuar con la ciudadanía (Guadián, 2011b). Su limitación de 140 caracteres por mensaje (o tuit) exige mucha síntesis.

En formato teletipo la ciudadanía puede tener en su perfil de Twitter un listado cronológico de las noticias publicadas por las personas e instituciones a las que siguen. Esas breves noticias pueden ser profundizadas en la página web institucional, el blog, o el medio de comunicación que inspiraron esos mensajes.

Las ventajas de las redes sociales son que permiten establecer un diálogo al mismo nivel con la ciudadanía; facilitan el intercambio de ideas y opiniones en un lenguaje sencillo, y permiten hacer llegar mensajes a la ciudadanía de forma proactiva en lugares en los que la ciudadanía está más cómoda. La ciudadanía no tiene que ir a la web municipal a ver las noticias relevantes, sino que esas noticias se publican en sus perfiles de las redes sociales.

Entre los inconvenientes, para las personas con responsabilidad política y pública estaría la necesidad de aprender nuevas formas de comunicación, crear y mantener su identidad digital con perfiles de las redes sociales (evitando suplantaciones de identi$\operatorname{dad}^{25}$ ). Para la ciudadanía, una vez más, las barreras tienen que ver con la brecha digital, personas que por distintos motivos no acceden a Internet, bien por el coste económico elevado del acceso, bien porque no quieren saber o no les apetece. Y entre las personas que acceden a Internet, habrá personas que, por decisión personal, optan por no estar en las redes sociales y personas que no tienen el tiempo, la motivación o

${ }^{23} \mathrm{http}: / /$ bit.ly/vDsHSj

${ }^{24} \mathrm{http}: / /$ bit.ly/9RuQqJ

${ }^{25}$ La suplantación de la identidad en Internet es una actividad maliciosa en la que un atacante se hace pasar por otra persona por distintos motivos. Un caso típico de suplantación de identidad es, en las redes sociales, crear un perfil de otra persona e interactuar con otros usuarios haciéndose pasar por ella. Información para saber cómo actuar en esos casos en la web de INTECO: http://bit.ly/iAW1O1 
los conocimientos básicos necesarios para crearse un perfil y participar en ellas. Una buena práctica, tanto a nivel personal como para la institución pública o cada una de sus áreas, servicios, programas, es la de reservar los espacios virtuales en todas las redes sociales y plataformas 2.0 más utilizadas y habituales. Se publique o no contenido en ellas, es una forma de evitar suplantaciones de identidad.

\subsection{Deliberación y colaboración}

En sociedades complejas donde los problemas a abordar desde las instituciones públicas pueden tener múltiples perspectivas, es importante contar con opiniones diversas para tener en cuenta las distintas posibilidades y alternativas de actuación; sopesar los pros y los contras; valorar las ventajas e inconvenientes; y, posteriormente, las personas con responsabilidad pública y/o política decidirán en base a su criterio.

Las TIC permiten recopilar de forma rápida opiniones, valoraciones, percepciones de los agentes implicados en una decisión política y pública. A continuación se muestran algunas herramientas que muestran cómo es posible trabajar colaborativamente para contar con opiniones diversas que permitan mejorar el proceso de elaboración y puesta en marcha de políticas y servicios públicos.

\subsubsection{Herramientas para trabajar colaborativamente}

Entre las plataformas de trabajo colaborativo más destacadas, se encuentran Google Apps, accesible desde Internet con cualquier navegador; Microsoft Office 365 o Zimbra, desarrollada con software libre. Estas plataformas ofrecen soluciones tecnológicas para las necesidades más comunes y habituales de nuestro trabajo diario (correo electrónico, agenda de contactos, calendario, gestor de tareas, chat, almacenamiento de documentación en la nube, publicación de contenido, redacción de documentos, hojas de cálculo...) (EUSKADINNOVA, 2010).

Google Apps cuenta con Google Drive, un paquete ofimático en Internet que permite crear documentos (de texto, hojas de cálculo, formularios, presentaciones, dibujos) privados o públicos como páginas web. Al mismo tiempo, permite compartir un documento con varias personas para que todas puedan editar el mismo documento de forma simultánea y en tiempo real.

Los proyectos "Adopta a un diputado" ${ }^{26}$ y \#adoptaunsenador, pretendían transcribir la información publicada en PDF sobre la declaración de bienes de representantes del Congreso y del Senado español. Mediante una sencilla hoja de cálculo colaborativa ${ }^{27}$ y en tan solo 4 días, diferentes personas transcribieron de manera totalmente voluntaria y descentralizada la información, utilizando una hoja de Google Drive. Los datos de esa hoja de cálculo se pueden consultar y reutilizar (Pro Bono Público, 2011).

Entre las ventajas de trabajar de forma colaborativa encontramos: la posibilidad de generar sinergias, evitando duplicidades y solapamientos; eliminar reuniones y

\footnotetext{
${ }^{26} \mathrm{http}: / /$ bit.ly/1cVWxIV

${ }^{27} \mathrm{http}: / /$ bit.ly/JV2Rqr
} 
desplazamientos; ahorrar recursos (económicos, personales, materiales); reducir los tiempos en la redacción incorporando en un mismo y único documento las propuestas de varias personas que trabajan sobre él.

Una condición importante para que el trabajo colaborativo sea efectivo es que haya una buena coordinación del trabajo y se establezcan unas pautas y reglas claras a la hora de trabajar. Los inconvenientes de esta nueva forma de trabajo son, sobre todo, culturales, por las dificultades para cambiar procedimientos de trabajo y mentalidades burocráticas sobre quién es competente para hacer una tarea, quién tiene la propiedad de determinada información o de quién es la autoría de un trabajo.

\subsubsection{Wikis}

Una wiki (en hawaiano, wiki wiki significa rápido) es una aplicación informática que permite que los documentos allí alojados (las páginas wiki) sean escritos de forma colaborativa a través de un navegador, siendo muy sencillo dar formato, crear enlaces, etc. Las páginas wiki pueden ser visitadas y editadas por cualquier persona, si son abiertas, o por las personas registradas, si son privadas. Cuando alguien edita una página wiki, sus cambios aparecen inmediatamente en la web.

Entre los usos de las páginas wikis para contar con la participación ciudadana, destacamos la iniciativa TallerWeb1.0, puesta en marcha en el año 2006 por la Asociación InternetyEuskadi del País Vasco (Ramilo, 2006). Su objetivo era contar con la ciudadanía para mejorar la presencia en Internet de las instituciones públicas y los servicios ofrecidos en sus páginas web. A través de la página wiki del TallerWeb1. $0^{28}$, en una fase virtual, una serie de colectivos, personas expertas y quienes lo desearon, publicaron sus comentarios e ideas de mejora sobre las páginas web de las instituciones vascas analizadas. Posteriormente, en una fase presencial un grupo de personas expertas analizó las cerca de 500 propuestas de mejora sobre las distintas web institucionales evaluadas y elaboraron un informe de mejora de cada una de las páginas web analizadas. Finalmente se definió el Decálogo de Vitoria-Gasteiz con una serie de recomendaciones para que las instituciones públicas mejorasen su presencia en Internet al servicio de la ciudadanía. Poco después la iniciativa se trasladó a Cataluña organizándose el TallerWeb2.0 (Ramilo, 2012).

El lema utilizado en el TallerWeb1.0 para animar a la participación de la ciudadanía fue "Veni, vidi, wiki", similar al "Veni, Vidi, Vici" de Julio César anunciando una victoria ante el senado romano en el año 47 a.C., pero con un matiz diferente y muy importante: ya no se trata de imponer o vencer; sino de colaborar y contribuir a mejorar un documento, una propuesta, una política o servicio público con las aportaciones de una pluralidad de personas que tienen sus propias percepciones de la realidad.

Entre las ventajas de usar estas plataformas estarían la sencillez y rapidez en la creación de contenidos, actualización, corrección, mejora y recuperación de versiones previas (historial); la creación de redes de trabajo aumentando la transparencia, la

\footnotetext{
${ }^{28} \mathrm{http}: / /$ bit.ly/ZPz9nk
} 
colaboración y la confianza entre las personas participantes; ahorro de tiempo y reducción de reuniones y viajes; ahorro de costes de mantenimiento y webmasters. Como inconvenientes, podrían encontrarse el cambio cultural de interiorizar una nueva forma de trabajo basada en compartir la información y construir el conocimiento de forma colaborativa.

\section{Ciudadanía y la sociedad civil en red}

Se están desarrollando aplicaciones fáciles de usar para que la ciudadanía informe a sus ayuntamientos de las incidencias en las calles (baches, graffitis, desperfectos en la vía, en el alumbrado, etc). El procedimiento es sencillo: se introduce el nombre de la calle en la web del servicio o en la aplicación para dispositivos móviles (smartphones o tabletas), se localiza el problema sobre un mapa, se explica y se envía la incidencia al ayuntamiento. Este sistema ayuda a resolver un problema que por otras vías tardaría más tiempo en localizarse y solucionarse. Algunos ejemplos pueden ser el pionero FixMyStreet ${ }^{29}$ para ciudades del Reino Unido, Verbeter De Buurt ${ }^{30}$ en Holanda o SeeClickFix ${ }^{31}$ en ciudades de Estados Unidos o Arreglamicalle.com y Otrobache.com en España (Alarcón, 2010).

Existen otras iniciativas como Change.org que es una comunidad en línea de personas y organizaciones que unen esfuerzos para ejercer presión contra las injusticias, creando peticiones y campañas que solicitan cambios deseables a gobiernos, empresas y otros actores importantes de la sociedad. Avaaz.org es una organización en la que un equipo de personas determina una campaña y se difunde entre sus miembros para que la firmen, sin que la ciudadanía pueda crear nuevas peticiones. MiFirma.com es otra iniciativa que permite recoger firmas mediante el uso del DNI electrónico para que éstas tengan validez jurídica.

Goteo.org es una red social de financiación colectiva (aportaciones monetarias) (crowdfunding) y colaboración distribuida (servicios, infraestructuras, microtareas y otros recursos) (crowdsourcing), desde donde impulsar el desarrollo autónomo de iniciativas con ADN abierto, que generasen nuevas oportunidades para la mejora constante de la sociedad y el enriquecimiento de los bienes y recursos comunes. En definitiva, una plataforma para la inversión de capital riesgo, en proyectos que contribuyan al desarrollo del procomún, el conocimiento libre y/o el código abierto basados en la inteligencia colectiva (Gutiérrez y Freire, 2013). Son numerosas las plataformas que están surgiendo con esta visión colaborativa. En este enlace tiene un listado muy completo: http://www.crowdacy.com/crowdfunding-espana/www.rodalia.info ofrece el estado de la red de Cercanías de Renfe en Barcelona a través de la información subida por las personas usuarias. Este tipo de iniciativas podrían trasladarse a otras áreas y servicios donde la colaboración ciudadana con las instituciones públicas es fundamental

\footnotetext{
${ }^{29}$ www.fixmystreet.com

${ }^{30}$ www.verbeterdebuurt.nl

${ }^{31}$ www.seeclickfix.com/citizens
} 
facilitando la toma de decisiones y la gestión eficaz y eficiente de los recursos. Las instituciones públicas deben entender que no están solas en la generación de valor público: hay múltiples actores igual de relevantes que quieren trabajar en red para la ciudadanía. La información de las instituciones públicas tiene valor oficial, pero nunca será tan rápida y valiosa como la aportada por la ciudadanía en el instante y desde lugar en el que se produce una incidencia (Estebaranz y Ramilo, 2013).

@ NieveVG, puesta en marcha en el año 2010 por la asociación ciudadana Colabora en Red para que la ciudadanía de Vitoria-Gasteiz pueda avisar, en tiempo real, a través de Twitter y utilizando el hashtag \#nievevg de situaciones en las que la nieve afecta a la movilidad por la ciudad. Desde la web de la iniciativa [http://nievevg.vecinosva.org], que recoge cronológicamente todos los incidentes, se podían enviar mensajes que se publicaban en el perfil de Twitter de @NieveVG. La etiqueta \#nievevg se utiliza como referencia en los perfiles institucionales del ayuntamiento relacionados con la nieve (@vg_elurranieve), la policía local (@vg_policia) y el perfil oficial de la ciudad (@vitoriagasteiz) (Estebaranz y Ramilo, 2013; Gatón, 2013).

\section{Reflexiones finales}

Este artículo ha hecho un repaso por algunas herramientas disponibles en Internet que pueden permitir a las instituciones públicas interactuar de una manera más dinámica, proactiva, eficiente, abierta o colaborativa con la ciudadanía. Las herramientas están disponibles. Pero es necesario superar las barreras mentales, organizativas y culturales que permitan aprovechar el potencial que ofrecen estas tecnologías.

Es fundamental que las instituciones públicas avancen desde planteamientos competenciales a visiones relacionales, con mentes, modelos organizativos, valores y actitudes abiertas, dinámicas, ágiles, sistémicas. Si no, seguirá utilizándose la tecnología para ahorrar desplazamientos presenciales, trasladándolos a la navegación por varias sedes electrónicas, sin aprovechar el potencial de las TIC para repensar y para transformar los procesos de elaboración de políticas públicas y de prestación de servicios poniendo a la ciudadanía en el centro de la acción pública, en lugar de seguir funcionando con una lógica competencial.

En este proceso de transformación, las tecnologías, sean del tipo que sean, pueden ser instrumentos que faciliten los cambios. Además de las ya conocidas TIC que permiten utilizar herramientas para mejorar la el acceso y difusión de la información y la comunicación, son interesantes otras tecnologías como las TAC, tecnologías para el aprendizaje y el conocimiento; las TEP, tecnologías para el empoderamiento y la participación; sin olvidar las TEC, tecnologías para la gestión de las emociones y la cooperación (Consorcio de Inteligencia Emocional, 2012; Reig, 2011).

Todas estas tecnologías pueden ser medios que contribuyan al fin último de conseguir que las instituciones públicas sean sociables y trabajen en red al servicio de (y junto con) la ciudadanía. Una vez más, es decisión humana usar de manera efectiva las mismas para aprovechar su potencial transformador en las personas y en las organizaciones. Quizá lo que para algunas personas lectoras pueda parecer a día de 
hoy Ciencia Ficción en sus organizaciones, en 30 años forme parte de la Ciencia de la Administración.

\section{Bibliografía}

Alarcón, G. (2010). "Mejorando la comunicación entre ciudadanía y Administración pública", en Blog Open Government. En: http://bit.ly/anzLox [Consultado $12 / 01 / 2014]$.

Barber, B. (2001). "Which technology for which democracy? Which democracy for which technology?", en International Journal of Communications Law and Policy, no 6 , pp. 1-8.

Borge, R., Colombo, C., y Welp, Y. (2008). "Análisis explicativo de la participación ciudadana electrónica y presencial en el ámbito municipal de Cataluña", en Revista de Internet, Derecho Y Política, $\mathrm{n}^{\circ}$ 6, pp. 13-31.

Bosco, R., y Caldana, S. (2012). "La web del Senado que costó 450.000 euros, reproducida a coste cero", en El País. Madrid. En: http://bit.ly/UYpjPc [Consultado $12 / 01 / 2014]$.

Consorcio de Inteligencia Emocional (2012). ¿Qué es el CIE?. En: http://bit.ly/Kf8iQv [Consultado 12/01/2014].

CORA. (2013). Informe de la Comisión para la Reforma de las Administraciones Públicas. Madrid. En: http://bit.ly/1hOguFT [Consultado 12/01/2014].

Criado, J. I., y Martínez, G. (2010). Blogging político y personalización de la democracia local en España y Portugal. Evidencias presentes y propuestas de futuro. Fundación Alternativas, Madrid. En: http://bit.ly/fIILvQ [Consultado 12/01/2014].

Criado, J. I., y Ramilo, M. C. (2001). "e-Administración: ¿Un reto o una nueva moda? Problemas y perspectivas de futuro en torno a Internet y las tecnologías de la información y la comunicación en las Administraciones públicas del siglo XXI", en Revista Vasca de Administración Pública, vol. 61, n 1, pp. 11-43.

Criado, J. I., Ramilo, M. C., y Salvador, M. (2002). "La Necesidad de Teoría(s) sobre Gobierno Electrónico. Una propuesta Integradora". Ensayo presentado en el "XVI Concurso de ensayos y Monografías sobre Reforma del Estado y Modernización de la Administración pública”, sobre Gobierno Electrónico del CLAD. Mención Honorífica.

Estebaranz, J., y Ramilo, M. C. (2013). "Administraciones públicas locales vascas y redes sociales, más allá de Irekia". Congreso GIGAPP. Madrid. En: http://bit.ly/JV54Cb [Consultado 12/01/2014].

EUSKADINNOVA. (2010). Programa, documentación y vídeos de la Jornada Soluciones para trabajar colaborativamente. Parque Tecnológico de San Sebastián. En: http://bit.ly/cGlDw2 [Consultado 12/01/2014].

F. Barrero, D., Criado, J. I., y Ramilo, M. C. (2006). "Política y Web 2.0", en III Congreso Online del Observatorio para la Cibersociedad, Barcelona. En: http://bit.ly/1ce7mm9 [Consultado 12/01/2014].

Gatón, I. (2013). "\#nievevg: una iniciativa ciudadana convertida en Trending Topic". en GasteizHoy.com. Vitoria-Gasteiz. En: http://bit.ly/YAhYEV [Consultado 12/01/2014]. 
Generalitat de Cataluña (2013). Guía de usos y estilo en las redes sociales de la Generalitat de Cataluña (6a edición, octubre 2013). Departamento de la Presidencia, Barcelona. En: http://bit.ly/wYqvm4 [Consultado 12/01/2014].

Gobierno Vasco (2012). Guía de usos y estilo en las redes sociales del Gobierno Vasco. Servicio Central de Publicaciones, Vitoria-Gasteiz. En: http://bit.ly/OhVy6w [Consultado 12/01/2014].

Graells, J., y Ramilo, M. (2013). Ciudadanía y administraciones en red. INAP y Netbiblo, Madrid. En: http://kcy.me/m6gj [Consultado 12/01/2014].

Guadián, C. (2011a). "Cómo mejorar la presencia digital de un ayuntamiento colaborativamente", en Blog K-Government. En: http://bit.ly/sBp4Ez [Consultado $12 / 01 / 2014]$.

Guadián, C. (2011b). "Ideas para utilizar Twitter en un Ayuntamiento", en Blog KGovernment. En: http://bit.ly/kcsbJU [Consultado 12/01/2014].

Guadián, C. (2012). "Mapa de la blogosfera pública", en Blog K-Government. En: http://bit.ly/HO9nc8 [Consultado 12/01/2014].

Gutiérrez, A., y Freire, J. (2013). Manifiesto Crowd. La empresa y la inteligencia de las multitudes. Laboratorio de Tendencias. En: http://www.laboratoriodetendencias.com [Consultado 12/01/2014].

Hale, M. L., Musso, J. A., y Weare, C. (1999). "Electronic Democracy and the Diffusion of Municipal Web Pages in California", en Administration \& Society, vol. 31, $\mathrm{n}^{\mathrm{o}} 1$, pp. 3-27.

Hofmann, A., Ramírez, A., y Bojórquez, J. A. (2012). La promesa del Gobierno Abierto. Itaip-Infodf, México. En: http://bit.ly/13mUBUs [Consultado 12/01/2014].

Junta de Castilla y León (2012). Guía de usos y estilo en las redes sociales de la Junta de Castilla y León. En: http://bit.ly/1ce7tOF [Consultado 12/01/2014].

Moraga, E. (2009). "Ciudadanos con conocimiento, ciudadanos en movimiento. Acceso, reutilización y visualización de la información pública: herramientas estratégicas para la participación informada", en Taller-seminario VISUALIZAR'09, Madrid. En: http://bit.ly/2w1Obo [Consultado 12/01/2014].

O'Reilly, T. (2005). What is Web 2.0. Design Patterns and Business Models for the Next Generation of Software. En: http://oreilly.com/web2/archive/what-is-web20.html [Consultado 12/01/2014].

OCDE (1987). Administration as Service: The Public as Client. OCDE, París. En: http://bit.ly/Y8SI8K [Consultado 12/01/2014].

ONTSI (2011). Estudio sobre el conocimiento y uso de las Redes Sociales en España. Ministerio de Industria, energía y Turismo, Madrid. En: http://bit.ly/utepE6 [Consultado 12/01/2014].

ONTSI (2013). La sociedad en red. Informe anual 2012. Ministerio de Industria, energía y Turismo, Madrid. En: http://bit.ly/L25OpQ [Consultado 12/01/2014].

Ortíz, I. (2009). "El cambio que necesita la Administración pública", en Administraciones en Red. En: http://bit.ly/1dpQAqq [Consultado 12/01/2014].

Pro Bono Público (2011). Transparencia con una hoja de cálculo: \#adoptaunsenador. En: http://bit.ly/VBYQ7P [Consultado 12/01/2014]. 
Ramilo, M. C. (2003). "Gobierno electrónico en la práctica: Experiencias de interés en la CAPV", en Revista Vasca de Administración Pública, no 67, pp. 329-354.

Ramilo, M. C. (2005). "Ciudadanía y la necesidad de una Administración en red", en Revista Vasca de Administración Pública, $\mathrm{n}^{\circ}$ 72, pp. 321-352.

Ramilo, M. C. (2006). "TallerWeb1.0. Una experiencia participativa de mejora de la presencia de las instituciones políticas y públicas en Internet". CD Oficial de Comunicaciones de IX Jornadas TECNIMAP. Sevilla. En: http://bit.ly/lajonf7 [Consultado 12/01/2014].

Ramilo, M. C. (2012). "Seis años después del TallerWeb1.0", en Blog Enredando. En: http://bit.ly/wCqTzA [Consultado12/01/2014].

Ramilo, M. C., y Fernández, R. (2012). Ciudadanía y Participación en la Sociedad de la Información y del Conocimiento. (Estudios/Working Papers No. WP-2012-21). GIGAPP, Madrid. En: http://kcy.me/m6h6 [Consultado 12/01/2014].

Reig, D. (2011). "TIC, TAC, TEP y el 15 de octubre", en Blog El Caparazón. En: http://bit.ly/Kf8vTO [Consultado 12/01/2014].

Reig, D. (2012). Socionomía. ¿Vas a perderte la revolución social? Ediciones Deusto, Bilbao. En: http://bit.ly/WNBr6F [Consultado 12/01/2014].

Salvador, M., Cortés, R., Sánchez, R., y Ferrer, L. (Eds.). (2004). Els ajuntaments de Cataluña a Internet. UPF, Barcelona. En: http://bit.ly/Akmvfw [Consultado $12 / 01 / 2014]$.

Subirats, J. (2011). Otra sociedad, ¿otra política? De "no nos representan" a la democracia de lo común. Icaria Editorial, Barcelona. En: http://bit.ly/LyIFrH [Consultado 12/01/2014].

White House (2009). Open Government Directive. En http://1.usa.gov/4sbQJk [Consultado 12/01/2014]. 